\title{
How Many Nurses do we Need-Standards or Pseudo Work Study?
}

John P. Wattis, Consultant in the Psychiatry of Old Age, St James's University Hospital, Leeds;

KeITH J. B. Rix, Senior Lecturer in Psychiatry, University of Leeds, Consultant Psychiatrist, St James's University

Hospital, Leeds and DAvid A. Collins, Senior Nurse (Psychiatry), St James's University Hospital, Leeds

In manufacturing industries, measuring productivity is relatively easy. In many service industries where the job is discrete (e.g. mowing a given area of grass or serving a number of cups of tea), work study methods are still easy to apply. In medicine and nursing where both the product and its process of 'manufacture' are hard to measure, things become more difficult. Nowhere is this more so than in psychiatry. Perhaps because of this, the standard often applied in the past has been professional judgement, and the Royal College of Psychiatrists, for example, has suggested appropriate levels of nurse staffing in psychiatry of old age. ${ }^{1}$ Such standards are now being questioned and an alternative is being propounded, at least in the Yorkshire Region, based loosely on work study methods. ${ }^{2,3}$ Psychiatry and psychiatric nursing have nothing to fear from work study, provided that acceptable standards of service are defined, and work study methods are properly applied. The first of these is partly a political task although it is to be hoped that the politicians would take guidance from patients, their relatives, and the appropriate professionals. Work study can ensure that such standards are achieved in the most economic way. It cannot set the standards. The authors are concerned because an exercise has recently been mounted throughout Yorkshire that purports to be a measure of nursing load, but which neither defines the standards of nursing care to be achieved, nor uses adequate methods to measure the work involved.

A development of the Sullivan-Boyle method $^{2}$ of measuring psychiatric nursing loads has been implemented across the Region despite the gravest reservations expressed by both medical and nursing staff as to the adequacy of this method. The Sullivan-Boyle method itself is derived from the Goddard method pioneered in two general hospitals in Yorkshire in the 1960s. Goddard used the concept of a standard 'unit' of nursing time calculated on the basis of detailed work study observation in the general wards of one hospital. He calculated that, for patients of average dependency, this unit would consist of 125 minutes per patient comprising 110 minutes clinical nursing time, 10 minutes administrative time, and five minutes domestic work, although he envisaged that the vast bulk of domestic work would be organised separately from nursing services. He provided a method for measuring dependency which could be used to adjust up or down the standard nursing unit. On this basis, and with adjustments for holidays, sick leave, etc., he was able to calculate the nursing staff levels needed on wards, and to check this 'experimentally', in another general hospital. He also suggested that nursing establish- ment should be calculated on the basis of the average bed occupancy which could lead to serious problems in units whose occupancy varies substantially, for example as a result of seasonal variations in the incidence of illness.

Although Goddard's methodology is adequate it cannot be stressed too much that he made no attempt to define what was a proper standard of nursing care. Furthermore, although Goddard expressed the view that "the amount of time needed in the care of the patient increases progressively with the physical dependency of the patient" he was careful to counsel against the application of this principle to psychiatric nursing: "It must be understood that this assumption refers only to general hospitals. In fields such as mental nursing this criterion need not necessarily apply." In spite of Goddard's note of caution, Sullivan and Boyle have applied his methodology to psychiatry and made a number of completely unwarranted assumptions.

First, and most important, no attempt was made to work study the psychiatric nursing process. The unit of nursing time for the average psychiatric patient is assumed to be 110 minutes - the sum of 50 minutes 'bedside' nursing and 60 minutes 'collective' nursing (the administrative and domestic elements being ignored). Sullivan and Boyle offer no justification for choosing 60 minutes as the unit of 'collective' nursing time, even though Goddard commented that the range of 'collective' nursing was from $\mathbf{3 6}$ minutes (chest investigation) to 83 minutes (female medical). In work study terms, this is the equivalent of arguing that the 'standard time' for planting out 100 square metres of bedding plants should be the same as the standard time for mowing 100 square metres of grass because both tasks fall under the general rubric of 'gardening'. It might happen coincidentally that this was true, but it is highly unlikely. The only adequate way of deriving a 'standard time' for a task is to work-study that task.

Sullivan and Boyle made no attempt to do this, which would have meant measuring the time nurses spend in talking and listening to the patients, calming and sitting with emotionally upset patients, counselling, group therapy, social skills training, rehabilitation and the other important aspects of psychiatric nursing work related to planning individualised care and the provision of communication and psychological support systems for the care givers.

Second, arbitrary scales of dependency were constructed or adapted, and, in conjunction with the spurious unit of nursing time, used to calculate nursing loads. The scales used allowed the allocation of psychiatric patients to one of five categories of dependency. Not only was there no inves- 
tigation of the validity and reliability of their scales, but the categories used have been erroneously equated with the five categories of Goddard's 'table of standards'. It was thus deduced that a total of 80 standard minutes of nursing care was required for a Category 1 psychiatric patient because, being rated as 'No untoward emotional response, behaviour stable: No impairment of self care activity', he was regarded as having identical nursing needs to the general hospital patient who 'requires bed made when unoccupied; can wash and dress himself; moves about ward independently, or in chair; can get up to meals; uses sanitary annexe, e.g. WC'. There was no justification for this assumption whatsoever.

Furthermore, no attempt was made to allow for the fact that lower levels of nursing staff lead to increasing patient disturbance and dependency. Nevertheless, in their study, Sullivan and Boyle purported to show that nursing staff levels in their unit were inadequate. We have little doubt that they were, in this case, correct. The unit they were studying was a large old asylum (Stanley Royd Hospital, Wakefield) which has very low costs, even for a unit which deals chiefly with long-stay patients.

Their scale, which made no attempt to define adequate standards of care, and which used a methodology at best based on inspired guesswork, was then taken up by the Yorkshire Regional Health Authority and, with minor modification, applied to psychiatric units across the region.

There have been major problems in implementation. There are separate scales for short/medium-stay wards, long-stay and long-stay wards for the elderly. There is no separate scale for acute psychiatric wards for elderly people, So, when the scales were applied in Leeds Eastern Health Authority, an acute psychiatric ward for the elderly had to be rated on a scale which was largely concerned with physical dependency whilst some of the patients were acutely psychotic, and others were undergoing active assessment and rehabilitation. On the other hand, elderly physically disabled patients on an acute psychiatric ward would have to be rated on a scale designed to measure acute psychosis. A ward in another district used chiefly for the rehabilitation of elderly psychiatric patients was rated as though it was an elderly long-stay ward.

The study method is also open to criticism on the grounds that it assumes that there is a linear relationship between dependency calculated on arbitrary scales and minutes of nursing time. This relationship is nowhere demonstrated, neither is it 'verified' by experiment in another hospital as was the Goddard principle. Indeed this assumption is challenged by the work of others which has identified a nonlinear relationship between patient dependency and the nursing time required. ${ }^{4.5,6}$ These studies identify a ratio of $1: 2: 5$ between the three dependency levels of self care, medium care and full care and the nursing time required.

The modified Sullivan-Boyle study was opposed before it was implemented on these and other grounds. Nevertheless, it was implemented, and the results not surprisingly suggest that both acute and long-stay wards are over-staffed. In one teaching hospital acute unit, if staff were reduced to the levels suggested, it would be impossible to take student nurses as there would not be enough supervision for them and for some of the time it would be difficult or impossible to maintain minimum legal standards of one trained nurse per ward. In this unit, admissions recently had to be restricted for two weeks because of nursing shortages. Yet these figures are being fed back to the Region, and the next time a complaint is made about nursing shortages the hospital may be told that the psychiatric unit is 'overstaffed'.

We recognise the need to ensure that we all are working as efficiently as possible, but we repudiate this amateurish application of inappropriate methodology. The only result of this survey has been to waste precious nursing time, and to demoralise already hard-pressed nurses. We would welcome a serious application of work-study methods to psychiatric nursing, provided that adequate standards of care were first defined. The current work being undertaken by the Royal College of Nursing to define a set of professional standards appropriate to the requirements of today's patients and care-givers could provide a substantial step forward in this direction. Work study methods could then do what they are intended to do; measure the most economic way of achieving those standards. Until then, we would prefer to rely on established professional methods.

\section{REFERENCES}

${ }^{1}$ Group por the Psychiatry of Old Age (1978) Memorandum on nurse staffing needs for the hospital service for the elderly mentally ill. Bulletin of the Royal College of Psychiatrists, January, 4-5.

${ }^{2}$ Leeds Regional Hospttal BoArd (1963) Work Measurement as a Basis for Calculating Nursing Establishments: An Analytical Study: Harrogate. Leeds RHB.

${ }^{3}$ WAKEFIELD AREA HEALth AUthordTY (1977) Evaluation of Patient Dependency and Assessment of Nurse Staffing Needs in a Large Hospital for the Mentally Ill.

4Oxpord Regional Hospital Board, Operations Research Unit (1967) Management of Nursing Care, Oxford RHB.

5Mulugan, B. (1974) Patient-Nurse Dependency and Workload Index, King's Fund Project Paper No 2 (2nd Ed.), London.

${ }^{6}$ Norwich, H. S. \& SENIOR, O. E. (1971) Determining nursing establishments, Nursing Times, 67, Occasional Papers pp. 17-20. 